\title{
Proof-of-principle that a decoy virus protects oncolytic measles virus against neutralizing antibodies
}

This article was published in the following Dove Press journal: Oncolytic Virotherapy

\author{
Chun $X u^{1,2, *}$ \\ Annika Verena Goß \\ Carmen Dorneburg' \\ Klaus-Michael Debatin' \\ Jiwu Wei ${ }^{2}$ \\ Christian Beltinger' \\ 'Department of Pediatrics and \\ Adolescent Medicine, Section of \\ Experimental Pediatric Oncology, \\ University Medical Center Ulm, Ulm, \\ Germany; ${ }^{2}$ jiangsu Key Laboratory of \\ Molecular Medicine, Medical School of \\ Nanjing University, China \\ *These authors contributed equally to \\ this work
}

Correspondence: Christian Beltinger Department of Pediatrics and Adolescent Medicine, Section Experimental Pediatric Oncology, University Medical Center Ulm, Eythstraße 24, 89075 Ulm, Germany Tel +49 73I 50057032

Fax +49 73I 50057042

Email christian.beltinger@uniklinik-ulm.de
Background: Attenuated oncolytic measles virus (OMV) is a promising antitumor agent in early-phase clinical trials. However, pre-existing immunity against measles might be a hurdle for OMV therapy.

Methods: OMV was inactivated with short-wavelength ultraviolet light (UV-C). Loss of replication and oncolytic activity of UV-inactivated OMV were confirmed by tissue culture infective dose $50\left(\mathrm{TCID}_{50}\right)$ assay using Vero cells and by flow cytometry using Jurkat cells. An enzyme-linked immunosorbent assay was performed to verify that UV-inactivated OMV remained antigenic. Different doses of UV-inactivated OMV were pre-cultured in media supplemented with measles immune serum. The mixture was transferred to Jurkat cells and active OMV was added. Active OMV-induced death of Jurkat cells was monitored by flow cytometry.

Results: UV-inactivation abrogates OMV replication while maintaining its antigenicity. UVinactivated OMV sequesters pre-existing anti-MV antibodies in Jurkat cell culture, thereby protecting active OMV from neutralization and preserving oncolytic activity.

Conclusion: We prove the principle that a non-replicating OMV can serve as a "decoy" for neutralizing anti-MV antibodies, thereby allowing antitumor activity of OMV.

Keywords: acute lymphoblastic leukemia, oncolytic measles virus, anti-measles antibodies, decoy for anti-measles antibodies

\section{Introduction}

Measles virus (MV) is a single-stranded, negative-sense RNA virus belonging to the genus Morbillivirus within the family Paramyxoviridae. Its hemagglutinin $(\mathrm{H})$ and fusion (F) envelope glycoproteins are essential for MV entry into host cells. ${ }^{1}$ During infection, the H protein binds to the MV cell surface receptors SLAM, ${ }^{2} \mathrm{CD} 46^{3}$ or Nectin $4,{ }^{4,5}$ followed by F protein-mediated cell fusion. ${ }^{6}$ Immunoglobulin G (IgG) antibodies against many epitopes localized along the whole length of the $\mathrm{H}$ protein neutralize $\mathrm{MV}$ infectivity. ${ }^{7,8}$ Attenuated oncolytic measles virus (OMV) preferentially replicates and spreads within tumors. CD46, identified as the receptor for OMV, ${ }^{3}$ is over-expressed on human solid tumor cells compared to their normal non-transformed cells. ${ }^{9}$ Many preclinical and early-phase clinical studies have demonstrated efficient OMV antitumor activity against solid tumors and multiple myeloma. ${ }^{10}$ However, systemic OMV therapy in cancer patients with protective anti-MV IgG titers is challenging. ${ }^{11}$ One approach to overcome this barrier is to protect the virus within carrier cells. ${ }^{11,12}$ Along this line, we have used endothelial progenitor cells. ${ }^{12}$ However, preparation and infection of carrier cells is elaborate and cells need to home efficiently to the tumor. Aptamers directly 
against neutralizing antibodies of vesicular stomatitis virus have been used, albeit with moderate efficacy. ${ }^{13}$

We hypothesized that MV proteins can act as "decoys" for neutralizing anti-MV antibodies, thus preventing neutralization of OMV. In this work, we provide first-time evidence for this notion.

\section{Materials and methods Cells}

Vero cells (American Type Culture Collection [ATCC], Manassas, VA, USA) were cultured in DMEM (Thermo Fisher Scientific, Life Technologies, Waltham, MA, USA) supplemented with 10\% fetal bovine serum (FBS; Thermo Fisher Scientific) and $2 \mathrm{mM}$ L-glutamine (Biochrom, Berlin, Germany). The human pediatric T-acute lymphoblastic leukemia (ALL) cell line Jurkat (DSMZ, Braunschweig, Germany) was cultured in RPMI 1640 medium (Thermo Fisher Scientific) supplemented with 10\% FBS, 2 mM L-glutamine, $100 \mathrm{U} / \mathrm{mL}$ penicillin and $100 \mathrm{ug} / \mathrm{mL}$ streptomycin (Thermo Fisher Scientific). Cells were incubated at $5 \% \mathrm{CO}_{2}$ in a humidified incubator at $37^{\circ} \mathrm{C}$.

\section{OMV}

The OMV encoding the human sodium iodide symporter measles virus (MV-NIS) downstream of the hemagglutinin gene was obtained as high-titer purified supernatant from Imanis Life Sciences (Rochester, MN, USA) and titrated by tissue culture infective dose $50\left(\mathrm{TCID}_{50}\right)$ assay.

For ultraviolet (UV)-inactivation, MV-NIS was irradiated with 0.5 Joule $/ \mathrm{cm}^{2}$ short-wavelength ultraviolet light (UV-C) within a sterile cell culture hood (Herasafe KS18, Thermo Fisher Scientific) for $0.5 \mathrm{~h}$. Inactivation was confirmed by $\mathrm{TCID}_{50}$ assay.

\section{$\mathrm{TCID}_{50}$ assay and assessment of UV- inactivation}

MV-NIS titer was quantified on Vero cells and calculated as TCID $_{50} / \mathrm{mL}$ according to the Spearman-Karber formula, as described. ${ }^{14}$

For assessment of UV-inactivation of MV-NIS, syncytia formation was determined by crystal violet staining. To this end, cells were covered with $35 \mu$ crystal violet solution (Sigma-Aldrich Co., St Louis, MO, USA) for 0.5 h. Stained cells were washed with double distilled (dd) $\mathrm{H}_{2} \mathrm{O}$, dried at room temperature (RT) and photographed.

\section{Human MV immune serum}

Serum with neutralizing anti-MV antibodies was prepared from whole blood of a healthy donor. Blood was kept for $0.5 \mathrm{~h}$ at RT. Clotted blood was centrifuged for $20 \mathrm{~min}$ at $2000 \times \mathrm{g}$. Serum was inactivated at $56^{\circ} \mathrm{C}$ for $0.5 \mathrm{~h}$ prior to storage at $-80^{\circ} \mathrm{C}$. The anti-MV antibody index was determined at the Institute of Virology (Ulm University, Ulm, Germany) according to their standards and judged to be protective.

\section{MV-NIS infection and cell kill}

To assess the effect of UV-inactivated or active MV-NIS on Jurkat cells in vitro, $4 \times 10^{4}$ cells were seeded in RPMI 1640 medium (Thermo Fisher Scientific) into a 96-well U-bottom plate and incubated with UV-inactivated MVNIS at indicated multiplicity of infection (MOI) values, with MV-NIS at an MOI of 1 or with buffer only (negative control), for $3 \mathrm{~h}$. Cells were further cultured in RPMI 1640 medium supplemented with $10 \%$ FBS, 2 mM L-glutamine, $100 \mathrm{U} / \mathrm{mL}$ penicillin and $100 \mathrm{ug} / \mathrm{mL}$ streptomycin (Thermo Fisher Scientific). Cell viability was measured at $72 \mathrm{~h}$ postinfection by forward scatter/side scatter (FSC/SSC) flow cytometry (FACS Calibur, BD, Franklin Lakes, NJ, USA). Data were analyzed with FlowJo software (Tree Star, Ashland, OR, USA).

To validate UV-inactivated MV-NIS as a decoy to capture human anti-MV antibodies in vitro, UV-inactivated MVNIS at indicated MOIs was pre-incubated with $2 \%$ measles immune serum in RPMI 1640 medium for $0.5 \mathrm{~h}$. MV-NIS at an MOI of 1 was then added and the mixture was transferred to $4 \times 10^{4}$ Jurkat cells seeded into a 96 -well U-bottom plate for $3 \mathrm{~h}$. Cells were further cultured in RPMI 1640 medium supplemented with $10 \%$ FBS, 2 mM L-glutamine, $100 \mathrm{U} /$ $\mathrm{mL}$ penicillin and $100 \mathrm{ug} / \mathrm{mL}$ streptomycin (Thermo Fisher Scientific), and cell viability was measured at $96 \mathrm{~h}$ postinfection, as described above.

\section{Anti-MV lgG enzyme-linked immunosorbent assay}

An anti-MV IgG enzyme-linked immunosorbent assay (ELISA) (LS-F10275; LifeSpan Biosciences, Seattle, WA, USA) was used to validate the antigenicity of UV-inactivated MV-NIS. UV-inactivated MV-NIS was diluted in the presence of $2 \%$ measles immune serum and pre-incubated for $0.5 \mathrm{~h}$ at RT. Samples were then transferred to an ELISA plate coated with MV antigens. The amount of anti-MV IgGs, initially not caught by the decoy virus, was estimated from binding to the immobilized MV antigens by optical density measurement at $450 \mathrm{~nm}$.

\section{Statistical analyses}

Data are presented as means with standard deviation. Statistical differences were evaluated by an unpaired two-tailed 
Student's $t$-test using Graph Pad Prism 6.0e software (La Jolla, CA, USA).

\section{Results}

\section{UV-inactivated MV-NIS can act as a decoy to protect active MV-NIS from neutralizing anti-measles antibodies}

First, we wanted to know whether UV-inactivation inhibits replication of MV-NIS, thus rendering the virus nononcolytic. Indeed, in comparison to the strong oncolytic effect of active MV-NIS in Vero cells, incubation with UV-inactivated MV-NIS did not induce syncytia formation (Figure 1A). These results proved that UV-inactivated MV-NIS had lost its replicative capacity. Accordingly, UV-inactivated MV-NIS was not oncolytic in Jurkat cells (Figure 1B). Supplementation of the culture medium with $2 \%$ measles immune serum only marginally decreased the viability of the Jurkat cells.

Given that UV-inactivation may also damage viral proteins important for antibody binding and may thus decrease the decoy function of UV-inactivated MV-NIS, we determined its antigenicity by employing an ELISA with immobilized MV antigens (Figure 2). Since MV-immune patients will have
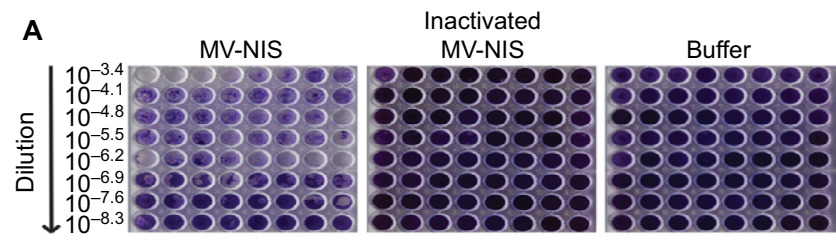

B

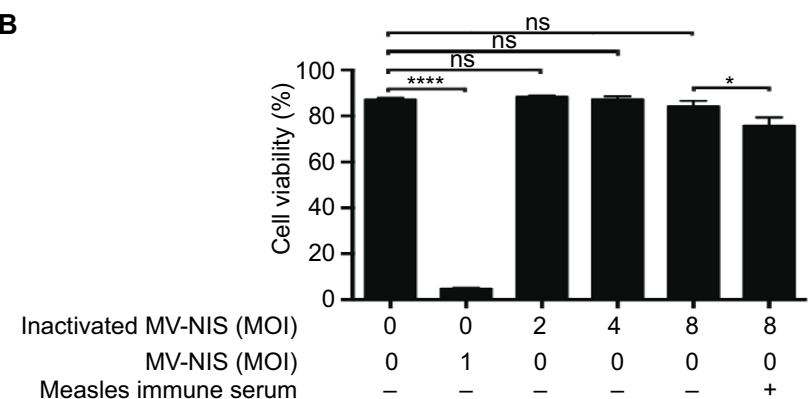

Figure I UV-inactivation abrogates replication of MV-NIS. MV-NIS was inactivated by 0.5 Joule/cm ${ }^{2}$ UV-C light for $0.5 \mathrm{~h}$.

Notes: (A) UV-inactivated MV-NIS is not oncolytic in Vero cells. Vero cells were incubated with dilutions of active MV-NIS, UV-inactivated MV-NIS or buffer and a $\mathrm{TCID}_{50}$ assay was performed. Syncytia formation was documented by using crystal violet staining after $6 \mathrm{~d}$. (B) UV-inactivated MV-NIS does not affect viability of Jurkat cells. Jurkat cells were incubated with buffer (negative control), MV-NIS (positive control) or UV-inactivated MV-NIS at indicated MOls in the presence or absence of $2 \%$ measles immune serum. Cell viability was determined after $72 \mathrm{~h}$ by flow cytometry using forward scatter/side scatter analysis. Results are means of triplicates. Statistical analysis was performed using a two-tailed unpaired Student's t-test. *p $<0.05$; **** $p<0.0001$.

Abbreviations: UV, ultraviolet; UV-C, short-wavelength ultraviolet light; MV-NIS, human sodium iodide symporter measles virus; $\mathrm{MOI}$, multiplicity of infection; ns, not significant; $\mathrm{TCID}_{50}$, tissue culture infective dose 50 . to be treated with the decoy prior to systemic administration of OMV, we aimed to mimic this clinical situation in vitro. To this end, UV-inactivated MV-NIS was serially diluted in the presence of $2 \%$ measles immune serum and pre-incubated for $0.5 \mathrm{~h}$ at RT before transfer into MV antigen-coated wells. For measles immune serum without UV-inactivated MV-NIS, an $\mathrm{OD}_{450}$ of 2.56, comparable to the technical positive control, was detected, proving strong binding of anti-MV IgGs to the MV antigens in the absence of the decoy virus (Figure 2). In stark contrast, a dose-dependent reduction of anti-MV IgGs to plate-bound MV antigens was seen for samples incubated with the decoy virus. This shows that the antigenicity of MVNIS is maintained after UV-inactivation.

Next, we investigated whether UV-inactivated MV-NIS can act as a decoy to sequester anti-MV antibodies, thus protecting active OMV from neutralization. Incubation of the T-ALL cell line Jurkat with active MV-NIS at an MOI of 1 induced complete cell death rapidly, within $72 \mathrm{~h}$ (Figure 3, left panel). To determine longer lasting protection, the effect of the decoy virus in protecting oncolytic virus from neutralization leading to decreased viability of Jurkat cells was determined after $96 \mathrm{~h}$. Indeed, viability of Jurkat cells significantly decreased, depending on the dose of the decoy virus given. However, the decoy virus, even at the highest dose, did not provide complete protection.

Taken together, we could prove the principle that MVNIS, rendered replication incompetent by UV-inactivation, can subsequently act as a decoy to protect active OMV from neutralizing antibodies.

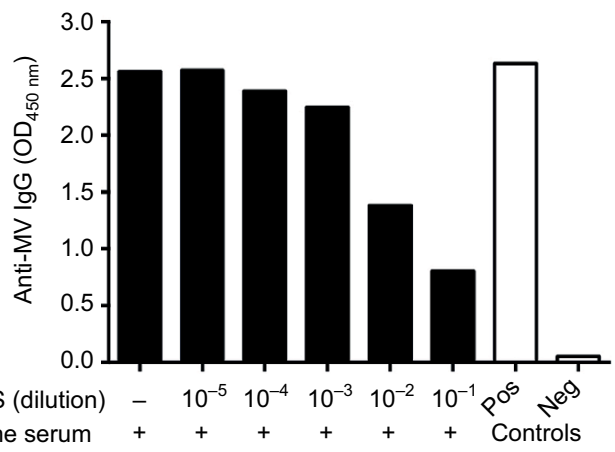

$\begin{array}{cccccccc}\text { Inactivated MV-NIS (dilution) } & - & 10^{-5} & 10^{-4} & 10^{-3} & 10^{-2} & 10^{-1} & 0^{5} \\ \text { Measles immune serum } & + & + & + & + & + & + & +\end{array}$

Figure 2 UV-inactivated MV-NIS remains antigenic.

Notes: MV-NIS was inactivated by 0.5 Joule $/ \mathrm{cm}^{2}$ UV-C light for $0.5 \mathrm{~h}$. UV-inactivated MV-NIS was serially diluted in the presence of $2 \%$ measles immune serum and preincubated for $0.5 \mathrm{~h}$ at room temperature. An ELISA was performed using plates coated with $M V$ antigens. Optical density at $450 \mathrm{~nm}\left(\mathrm{OD}_{450}\right)$ was measured to determine the amount of anti-MV IgGs that were initially not caught by the decoy virus and could therefore bind to the coated MV antigens. Technical positive and negative controls were run in parallel.

Abbreviations: ELISA, enzyme-linked immunosorbent assay; UV, ultraviolet; UV-C, short-wavelength ultraviolet light; MV-NIS, human sodium iodide symporter measles virus; IgG, immunoglobulin G; OD, optical density; Pos, positive; Neg, negative. 


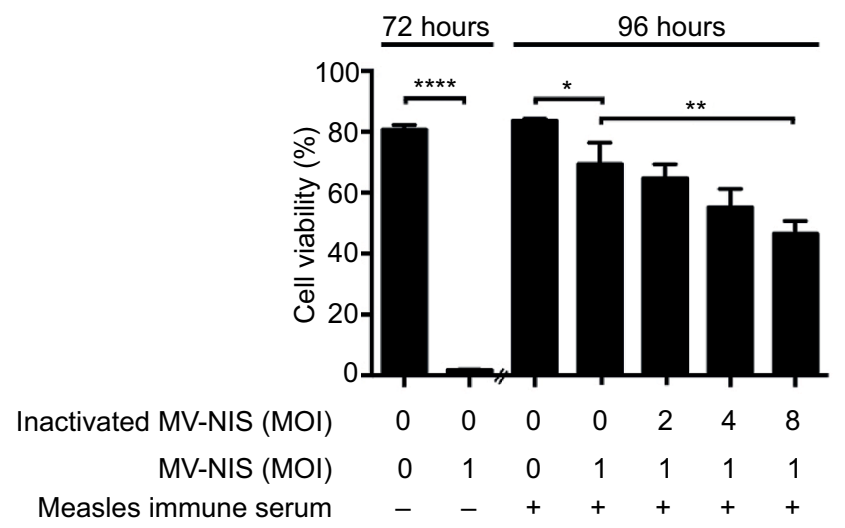

Figure 3 UV-inactivated measles virus protects against neutralization of active MVNIS by measles antibodies in vitro.

Notes: Jurkat cells were incubated with buffer (negative control) or with MV-NIS at an $\mathrm{MOI}$ of I. Cell viability was detected after $72 \mathrm{~h}$ by flow cytometry using forward scatter/side scatter analysis (first two bars). UV-inactivated MV-NIS at the indicated MOls was pre-incubated with medium containing $2 \%$ measles immune serum for 0.5 h. MV-NIS at an MOI of I was then added and the mixture was transferred to Jurkat cells for $3 \mathrm{~h}$. Cell viability was measured after $96 \mathrm{~h}$ (remainder of bars). Results are means of triplicates. Experiments were performed at least twice, with similar results. Statistical analysis was performed using a two-tailed unpaired Student's $t$-test. $* p<0.05$; ** $p<0.01$; **** $p<0.0001$.

Abbreviations: UV, ultraviolet; MV-NIS, human sodium iodide symporter measles virus; $\mathrm{MOI}$, multiplicity of infection.

\section{Discussion}

We have proved the principle that OMV rendered replicationincompetent while maintaining its antigenicity is a decoy for neutralizing anti-MV antibodies, thus protecting active OMV from neutralization and enabling them to kill cancer cells in vitro.

Several aspects and potential limitations of this study warrant consideration. First, the data on preserving antigenicity contrast with some publications that describe a loss of viral antigenicity post UV-inactivation. ${ }^{15-17}$ This can be explained by differences in radiation settings and differential sensitivity of the envelopes of different virus species. Second, the decoy did not provide complete protection against neutralizing antibodies. This may have been due to insufficient dosing or to incomplete preservation of antigenicity of the decoy. Third, it remains to be shown that the decoy approach also works in vivo.

Several conceptual aspects of a decoy approach call for a critical discussion. First, injection of decoy MV-NIS will boost MV immunity in patients, prohibiting multiple injections of active MV-NIS. However, "single-shot therapy" with MV-NIS may suffice to control cancer and boosting can be prevented by concurrent administration of cyclophosphamide, as recently shown for MV-NIS therapy of multiple myeloma. ${ }^{18}$ Furthermore, boosting the immune response may also have advantages, as antibody-dependent cellular phagocytosis and cytotoxicity (ADCP and ADCC) may be induced that could enhance the antitumor effect of the virus. ${ }^{19,20}$ Second, a dose- limiting side effect of the decoy approach could be immune complex diseases, such as vasculitis, glomerulonephritis or arthritis. However, there is very limited evidence for immunecomplex-mediated hypersensitivity in natural MV infection and there is no evidence in multiple myeloma patients treated intravenously with MV-NIS. ${ }^{18}$ Nevertheless, pre-existing immune complex-mediated diseases should be considered a contraindication for the decoy approach. However, due to the overall rarity of such diseases, especially in children, this issue would not significantly decrease the number of patients eligible for decoys. Third, it may be questioned whether decoys are as effective at sequestering antibodies resulting from natural infection as from vaccination. However, long-lasting measles immunity in both cases is predominantly mediated by antiMV antibodies of the IgG1 subclass. Finally, as alternatives to decoy viruses, recombinant $\mathrm{H}$ proteins or aptamers ${ }^{13}$ can be considered to sequester neutralizing antibodies.

In conclusion, we have provided an in vitro proof-ofprinciple that decoy OMV can sequester antibodies against OMV. This warrants future in vivo studies of this principle. The decoy approach may also be applicable for viruses other than OMV.

\section{Acknowledgments}

This work was supported in part by a grant from the Deutsche Forschungsgemeinschaft (DFG) to $\mathrm{CB}$; $\mathrm{CX}$ was funded by a grant from the China Scholarship Foundation; $\mathrm{AVG}$ is a $\mathrm{PhD}$ student within the International Graduate School in Molecular Medicine, Ulm, that is funded by the DFG.

\section{Disclosure}

The authors report no conflicts of interest in this work.

\section{References}

1. Wild TF, Malvoisin E, Buckland R. Measles virus: both the haemagglutinin and fusion glycoproteins are required for fusion. $J$ Gen Virol. 1991;72(Pt 2):439-442.

2. Tatsuo H, Ono N, Tanaka K, Yanagi Y. SLAM (CDw150) is a cellular receptor for measles virus. Nature. 2000;406(6798):893-897.

3. Dörig RE, Marcil A, Chopra A, Richardson CD. The human CD46 molecule is a receptor for measles virus (Edmonston strain). Cell. 1993;75(2):295-305.

4. Mühlebach MD, Mateo M, Sinn PL, et al. Adherens junction protein nectin-4 is the epithelial receptor for measles virus. Nature. 2011;480(7378):530-533.

5. Noyce RS, Bondre DG, Ha MN, et al. Tumor cell marker PVRL4 (nectin 4) is an epithelial cell receptor for measles virus. PLoS Pathog. 2011;7(8):e1002240.

6. Malvoisin E, Wild TF. Measles virus glycoproteins: studies on the structure and interaction of the haemagglutinin and fusion proteins. J Gen Virol. 1993;74(Pt 11):2365-2372.

7. de Swart RL, Yüksel S, Osterhaus AD. Relative contributions of measles virus hemagglutinin- and fusion protein-specific serum antibodies to virus neutralization. $J$ Virol. 2005;79(17):11547-11551. 
8. Bouche FB, Ertl OT, Muller CP. Neutralizing B cell response in measles. Viral Immunol. 2002;15(3):451-471.

9. Anderson BD, Nakamura T, Russell SJ, Peng KW. High CD46 receptor density determines preferential killing of tumor cells by oncolytic measles virus. Cancer Res. 2004;64(14):4919-4926.

10. Robinson S, Galanis E. Potential and clinical translation of oncolytic measles viruses. Expert Opin Biol Ther. 2017;17(3):353-363.

11. Castleton A, Dey A, Beaton B, et al. Human mesenchymal stromal cells deliver systemic oncolytic measles virus to treat acute lymphoblastic leukemia in the presence of humoral immunity. Blood. 2014;123(9): 1327-1335.

12. Wei J, Wahl J, Nakamura T, et al. Targeted release of oncolytic measles virus by blood outgrowth endothelial cells in situ inhibits orthotopic gliomas. Gene Ther. 2007;14(22):1573-1586.

13. Muharemagic D, Zamay A, Ghobadloo SM, et al. Aptamer-facilitated protection of oncolytic virus from neutralizing antibodies. Mol Ther Nucleic Acids. 2014;3:e167.

14. Langfield KK, Walker HJ, Gregory LC, Federspiel MJ. Manufacture of measles viruses. Mol Biol. 2011;737:345-366.

15. Phillips BA. In vitro assembly of polioviruses. I. Kinetics of the assembly of empty capsids and the role of extracts from infected cells Virology. 1969;39(4):811-821.
16. Furuya Y, Regner M, Lobigs M, Koskinen A, Müllbacher A, Alsharifi M. Effect of inactivation method on the cross-protective immunity induced by whole 'killed' influenza A viruses and commercial vaccine preparations. J Gen Virol. 2010;91(Pt 6):1450-1460.

17. Fan YC, Chiu HC, Chen LK, Chang GJ, Chiou SS. Formalin Inactivation of Japanese encephalitis virus vaccine alters the antigenicity and immunogenicity of a neutralization epitope in envelope protein domain III. PLoS Negl Trop Dis. 2015;9(10):e0004167.

18. Dispenzieri A, Tong C, LaPlant B, et al. Phase I trial of systemic administration of Edmonston strain of measles virus genetically engineered to express the sodium iodide symporter in patients with recurrent or refractory multiple myeloma. Leukemia. 2017;31(12):2791-2798.

19. Watanabe M, Wallace PK, Keler T, Deo YM, Akewanlop C, Hayes DF. Antibody dependent cellular phagocytosis (ADCP) and antibody dependent cellular cytotoxicity (ADCC) of breast cancer cells mediated by bispecific antibody, MDX-210. Breast Cancer Res Treat. 1999;53(3):199-207.

20. Rajasekaran N, Chester C, Yonezawa A, Zhao X, Kohrt HE. Enhancement of antibody-dependent cell mediated cytotoxicity: a new era in cancer treatment. Immunotargets Ther. 2015;4:91-100.

\section{Oncolytic Virotherapy}

\section{Publish your work in this journal}

Oncolytic Virotherapy is an international, peer-reviewed, open access online journal publishing original research, study protocols, reviews, editorials and commentaries on all aspects of oncolytic virology, namely the application of oncolytic viruses for the treatment of cancer. Specific topics in the journal include: Rationale and theoretical aspects of oncolytic virotherapy including in vitro, in vivo and mathematical

\section{Dovepress}

modeling; and practical application and problem solving in the clinic including identification of potential responders through biomarkers and genetic profiling. The manuscript management system is completely online and includes a very quick and fair peer-review system, which is all easy to use. Visit http://www.dovepress.com/ testimonials.php to read real quotes from published authors. 